\title{
A Tool to Improve Communicable Disease Surveillance Data
}

\author{
Candace M. Noonan-Toly ${ }^{\star 1}$, Charles DiDonato ${ }^{2}$ and Hwa-Gan Chang ${ }^{1}$
}

'Epidemiology, NYS Department of Health, Albany, NY, USA; ${ }^{2}$ NTT Data, Albany, NY, USA

\section{Objective}

Improved methods for user analysis of communicable disease surveillance data in New York State (NYS), excluding New York City (NYC).

\section{Introduction}

NYS (excluding NYC) has a very robust Communicable Disease Electronic Surveillance System (CDESS). This system provides disease specific modules, as well as a tracking system for contacts, and a perinatal infant tracking system. This system provides an easy way for users to quickly download a file with all of their data.

NYS (excluding NYC) tracks, on average, 300 infants of hepatitis B surface antigen (HBsAg) positive mothers annually. CDESS provides an infant tracking module for local health departments (LHDs) to enter and monitor vaccine information, add multiple infants per mother, and track patient movement and loss to follow-up. The tool allows LHDs to analyze infants' data by birth year cohort, with all of their current vaccination and serology information available in one record.

In 2013 and 2014, more than 13,000 cases of gonorrhea were reported to CDESS in NYS (excluding NYC). From November 2013 through May 2014, only $61 \%$ of cases were adequately treated with a regimen recommended by the Centers for Disease Control and Prevention (CDC) STD Treatment Guidelines for Gonorrhea ${ }^{1}$, and $29 \%$ were missing treatment information. The CDESS system allows the LHDs to track patients who have inadequate and/or missing treatment information.

\section{Methods}

The infant's birth information, mother's information, and the provider information in CDESS are prepopulated from newborn screening for each infant born to an HBsAg positive mother. LHDs enter the infant's vaccination record and the infant's serology record. To access the infant's data on CDESS, the LHD needs to know the mother's information: either the unique identifier or complete name and date of birth.

Gonorrhea investigations are automatically created by CDESS by data from the Electronic Clinical Laboratory Reporting System (ECLRS). Demographic, provider, and laboratory information is prepopulated by ECLRS. LHDs create the case upon review and perform follow-up and update the case supplemental form with information regarding risk, treatment, and additional provider data.

The CDESS data resides in Oracle tables. Using SAS V9.3, the complex relational data is collated into one record per infant for the perinatal data, and one record per patient for the gonorrhea data. This data is then converted to one comma separated values (CSV) file for each county and birth year cohort for perinatal, and county and case year for gonorrhea. These CSV files are pushed to the Oracle database by using the Oracle SQL*Loader utility. This loads the CSV files as a Character Large Object (CLOB) into an Oracle table in the CDESS database.

SAS runs automatically every night and sends the updated CSV files to CDESS. The updated CSV files are immediately available for download by the LHDs. Using these files county users can filter their data by provider, treatment, vaccine dosage, and age to be more proactive in protecting the health of New Yorkers.

\section{Results}

The simplicity of accessing one file removes multiple steps in finding the perinatal infants' vaccination and serology information. Using these CSV files, we can easily see that there are 259 babies currently being followed in the 2014 birth cohort. Twenty babies of the original 285 birth cohort moved out of jurisdiction, while six cannot be located. Of the 259, five did not receive HBIG at birth and four did not receive their first dose at birth. By eight months of age, $219(85 \%)$ had received three Hepatitis B vaccinations. Thirty-eight percent have already had their PVST.

Using the gonorrhea files, the LHDs can assess who is in need of treatment. From November 2014 through May 2015, 92\% of cases received adequate treatment, with only $2.6 \%$ missing data. Twenty percent fewer patients were considered to be inadequately treated because they received only one treatment in 2014-2015 than in 2013-2014.

\section{Conclusions}

Removal of multiple steps in data retrieval saves time for the LHDs. The LHDs now have an easy way to analyze their own data and be more proactive on their follow-up with physicians and families. All infants and patients within a jurisdiction can be monitored simultaneously. User-friendly systems and simple data analysis processes improve the overall quality of data collection.

\section{Keywords}

surveillance; communicable disease; gonorrhea treatment; perinatal hepatitis tracking

\section{References}

${ }^{1}$ Centers for Disease Control and Prevention. Update to CDC's Sexually Transmitted Diseases Treatment Guidelines 2010: Oral Cephalosporins No Longer a Recommended Treatment for Gonococcal Infections. MMWR 2012;61(31): 590-94.

\section{*Candace M. Noonan-Toly}

E-mail: candace.noonan-toly@health.ny.gov 